\title{
Toward a 10,000-element B-Mode Experiment
}

\section{Chao-Lin $\mathrm{Kuo}^{1}$ for the BICEP3 and POLAR1 Collaborations}

\author{
${ }^{1}$ Dept. of Physics, Stanford University \\ Kavli Institute for Particle Astrophysics and Cosmology \\ 385 Via Pueblo Mall, Stanford, CA94305, USA \\ email: clkuo@stanford.edu
}

\begin{abstract}
In this paper, we introduce two compact, large-throughput CMB polarimeter designs (POLAR1 and BICEP3). These pathfinder experiments will pave the way for a comprehensive multi-frequency South Pole B-mode survey that, when jointly analyzed with arcminute-scale polarization data, can conclusively answer the question whether there is an appreciable fraction $(>1 \%)$ of the primordial perturbations in the form of tensor modes (gravitational waves).
\end{abstract}

Keywords. Cosmology: cosmic microwave background, instrumentation: polarimeters

\section{Introduction}

Over the past year, several CMB polarization experiments targeting B-modes started observation with approximately a thousand detectors. Historically, the CMB community tends to be overly optimistic when it comes to predicting the performance of the experiments. However, based on the achieved sensitivity improvements over previous projects that had published limits on $r$, it is quite reasonable to expect the ongoing experiments to reach $r \equiv T / S=0.02 \sim 0.05$ (Table 1.). This range corresponds to the expected level of Galactic foregrounds in the cleanest part of the sky at the optimal frequency. A few other experiments that will soon be operational should also be able to reach a similar sensitivity.

The next phase of CMB polarization research is about following up these measurements both in sensitivity and in frequency coverage. For the current generation of experiments, the combination of foregrounds, lensing, and potentially instrument systematics will likely limit the detectable $r$ to $>0.05$. This is especially true if we adopt the widely accepted detection threshold of 5- $\sigma$ in elementary particle physics. Such a standard would be very appropriate for the discovery of primordial gravitational waves, an extraordinary claim in physics of fundamental interactions.

To make further progress, the main challenge is still the overall survey speed. The current CMB experiments are nearly background-limited and fully utilizing the available throughput in the optical systems. Following up these experiments would require expanding the frequency range for each focal plane element (multichroic) and/or increasing the total throughput. The former is a technical challenge that many groups are attempting. The latter strategy, which will be discussed in this article, is more straightforward and can also be combined with multichroic focal plane technologies when they become mature in the future.

\section{Dealing with Lensing: Two Approaches}

The lensing-induced B-mode signal is proportional to $\sim \ell^{2}$ at low multipoles, with a power equivalent to $r=0.0255$ at $\ell=85$. Based on Table 1 , the ongoing experiments will start to hit lensing after a few years of observation. To make further progress on 
Table 1. Past and Ongoing B-mode Experiments

\begin{tabular}{|c|c|c|c|c|c|c|c|c|}
\hline Experiment & NET & $\boldsymbol{\Gamma}(\mu \mathbf{K}-\sqrt{s}$ & ) $1 \mathrm{yrs}$ & collectec & d |yrs & expectec & $\mid r-1$ & $-\operatorname{limit}(95 \%)$ \\
\hline | BICEP & & 55 & | & 3 & | & 3 & | & 0.72 (2 yrs) \\
\hline QUIET-Q & & 69 & | & 0.7 & | & 0.7 & | & 2.2 \\
\hline QUIET-W & & 87 & 1 & 1.3 & 1 & 1.3 & | & 2.8 \\
\hline | BICEP2 & & 15.9 & I & 2.5 & 1 & 3 & 1 & $?$ \\
\hline Keck Array & & 11.5 & 1 & 1.5 & 1 & 4.5 & 1 & $?$ \\
\hline SPTPOL & & $\sim 16$ & I & 0.5 & I & 4 & 1 & $?$ \\
\hline POLARBEAR | & & $\sim 19$ & I & 0.5 & 1 & 3 & | & $?$ \\
\hline
\end{tabular}

$r$, there are two complementary approaches. The first approach is to increase the sky coverage and remove lensing in the power spectrum in the same way instrumental noise is debiased in temperature power spectrum measurements. After Planck, the expected amount of lensing will be determined to better than $3 \%$, simply due to the fact that all cosmological parameters, as well as the E-mode polarization, will be very well determined. The measured B-modes at $\ell \sim 100$ can be compared with this expected level and the significance for excess B-modes can be calculated.

Alternatively, the lensing deflection field can be reconstructed from arcminute-scale B-mode measurements. The expected lensing contribution to degree-scale B-modes can be derived by the mathematical "lensing" of the observed E-polarization using the reconstructed deflection field, which can then be subtracted from the observed B-map. This delensing procedure can be iterated to achieve a better removal fraction.

These two approaches are complementary because very different assumptions are made in the lensing removal process. It would be a powerful confirmation if the same conclusion on $r$ is deduced using two methods. The first method especially puts a premium on overall system sensitivity. By increasing the sky coverage, the sensitivity to primordial B-modes continues to improve as $f_{s k y}^{-1 / 2}$ until the clean part of the sky runs out. At South Pole the accessible clean sky is approximately 3000-4000 square degrees.

\section{Compact Large-Throughput CMB Experiments}

The primordial tensor-induced B-mode signal peaks at $\ell \sim 100$, corresponding to an angular scale of a few degrees. A $30 \mathrm{~cm}$ aperture telescope operating at $150 \mathrm{GHz}$ has a FWHM of $30^{\prime}$, which is more than sufficient to resolve the primordial B-polarization. This suggests that if systematic effects are under control, it is advantageous to pack a given number of detectors into optical systems that are as compact as possible, since the total cost of the experiment is a sharp function of the size and weight of the optics. Another very significant advantage for compact optical systems is that they allow active rotation of the entire instrument. As has been demonstrated in BICEP, QUaD and QUIET, redundant polarization measurements at multiple instrument angles (especially pairs separated by $180^{\circ}$ ) are crucial for distinguishing instrumental effects from polarization signals from the sky. This systematics mitigation technique cannot be overemphasized.

In this section, we discuss two compact optical designs with very large throughputs: the crossed-Dragone reflectors and the refractive telescopes. 
Crossed-Dragone Reflectors

A crossed-Dragone telescope consists of two off-axis, lightly curved reflectors. In studies of CMB telescope optics, crossed-Dragone emerges as the front running design due to its superior field-of-view and low polarization systematics. Because of its overwhelming advantages, a similar optical design is being considered for the NASA-CMBPOL mission concept "EPIC-IM".

A crossed-Dragone has no intermediate stop and one must therefore control where the spillover goes. The simplest solution is to use random diffusive surfaces to scatter the spillover onto the cold sky (15-20K at the South Pole). Such surfaces can be used to cover the inside of an optical enclosure that completely surrounds both reflectors (Figure 1a). Full non-sequential ray tracing simulations indicate that on average it takes $\sim 3$ reflections for a photon to escape the enclosure and evenly distribute on the sky. Using low-emissivity surfaces the loading from the spillover will be negligible. A reflective Winston cone ground shield will limit the diffuse sidelobes to within $30^{\circ}$ of the boresight direction and away from the Galaxy and the ground.

A 1.6-m crossed-Dragone telescope, known as POLAR1, is being developed to test these novel ideas. If the spillover termination scheme is demonstrated, such design would clearly be a very economical solution for the next generation ground-based lensing machine, dubbed POLAR Array, which aims to survey a significant fraction of the sky to a few $\mu \mathrm{K}$ depth. The lensless design also greatly simplifies broadband anti-reflection coating, making it an ideal platform for future multichroic CMB receivers.

Because of the resolution advantage compared to refractors, crossed-Dragone reflectors can also play an important role in synchrotron monitoring at lower frequencies, in the context of tensor-mode searches. At $44 \mathrm{GHz}$, POLAR1's 1.6-m optics provides a FWHM well-matched to those from compact $(30-60 \mathrm{~cm})$ refractors at $100-150 \mathrm{GHz}$. With a similar spillover termination technique, the reflector can feed $\sim 180$ polarimeter pairs at Q-band, compared with QUIET's 19 polarimeters.

\section{Refractors}

The use of refractors for CMB started with BICEP (2005-2008), followed by BICEP2 (2010-) and Keck Array (2011-). These experiments are described in detail in Bischoff et al. and Pryke et al. in these proceedings. The optical elements are entirely contained in
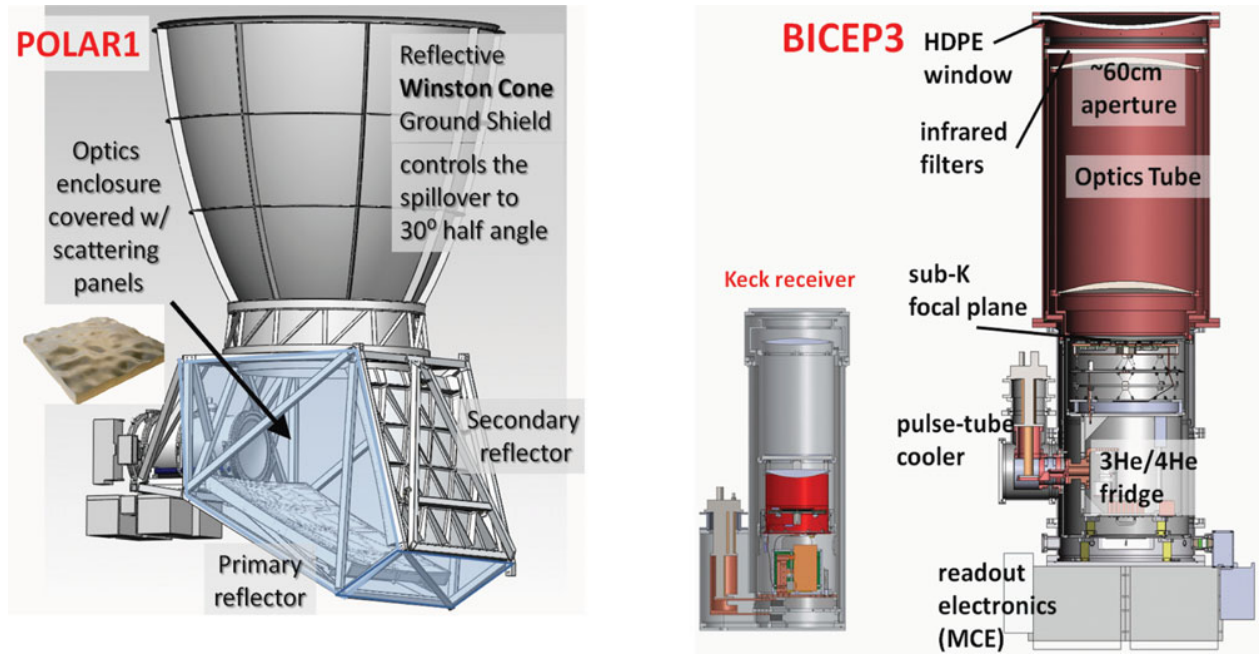

Figure 1. Left: POLAR1, a crossed-Dragone reflector with spillover scattered toward the sky. Right: BICEP3, a 60-cm cold refractor shown next to a Keck receiver for comparison. 
receiver cryostats, which provide stable, low-loading conditions, and a convenient optical cold stop. Even a simple two-lens refractor can provide superb throughput. Keck Array now has over 1,200 polarimeter pairs fed by 5 separate cryogenic 30 -cm refractors at the South Pole.

The Keck Array does require substantial logistic resources for a remote site in Antarctica. Each of the 5 receiver cryostats is cooled by its own water/glycol-chilled Pulse Tube cooler, consuming over $10 \mathrm{~kW}$ per receiver. Managing a large number of compressor Helium lines through the azimuthal cable wrap also proved to be a complicated task. Finally, each cryostat needs its own closed-cycle Helium-3 refrigerator, now becoming increasingly expensive. While these difficulties have all been surmounted in Keck Array, it is desirable to increase the number of detectors in each cryogenic system for the future.

The most straightforward solution is to simply increase the aperture size of the optics from BICEP's 30-cm. Figure 1b shows a 60-cm B-mode refractor, known as BICEP3, next to a Keck receiver for scale. At $150 \mathrm{GHz}$, this single 60-cm, f/1.6 two-lens system can feed $\sim 2,0002 f \lambda$ polarimeter pairs on the $44 \mathrm{~cm}$-dia. focal plane. The cryogenic challenge associated with a larger window can now be solved with large-aperture reflective mesh filters that we have successfully developed. The optical throughput of BICEP3 is as large as SPT-3G, yet it fits comfortably in the original BICEP mount. A BICEP3 proposal will be submitted to NSF for 2013/2014 deployment. Adding BICEP3 would more than double the throughput of the BICEP/Keck program. In addition, lessons learned from BICEP3 will be valuable for future expansion plans.

\section{A Focused Tensor-mode Program at the South Pole}

CMB at the South Pole has a long and successful history. Exciting new data are expected from the ongoing SPT and Keck Array. Benson et al. (in these proceedings) describes an ambitious plan (SPT-3G) to upgrade the SPT polarization receiver. This will provide excellent arcminute-scale polarization measurements over 2,500 square degrees of sky. This data will supply deflection measurements, and push SZ-cluster science, reionization, and CMB lensing cosmology to a new regime.

Looking forward, continuing the search for B-mode polarization generated from primordial tensor modes remains the most compelling science goal. The program described in this paper emphasizes a very focused attack on the searches for the primordial B-modes using compact, large-throughput optical systems. A comprehensive South Pole-based B-mode initiative consisting of a combination of $1.6-\mathrm{m}$ reflectors and $60-\mathrm{cm}$ refractors spanning a wide frequency range of $40-220 \mathrm{GHz}$ will greatly improve the expected measurements on $r$. By itself, this program will perform a deep search for tensor modes using the power spectrum-based lensing subtraction. When delensed by the deflection-field measured by SPT-3G, these measurements can provide a thorough, and likely conclusive search for inflationary primordial gravitational waves from the ground, reaching a 1- $\sigma$ uncertainty on $r$ well below 0.01 , or, in a more optimistic scenario, a high signal-to-noise detection!

\section{References}

Bischoff, C. et al. 2012, These Proceedings. Pryke, C. et al. 2012, These Proceedings.

Benson, B. et al. 2012, These Proceedings. 